EDITORIAL

\title{
POINT-OF-CARE TESTING (POCT): IMPORTANCE IN EMERGENCY MEDICAL /HEALTH CARE
}

\author{
ASM Giasuddin \\ Medical Research Unit (MRU), Medical College for Women \& Hospital \\ (MCW\&H), Medical and Health Welfare Trust, Uttara, Dhaka
}

Activities in Laboratory Medicine include teaching, research, administration, etc; however diagnostic laboratory service should be given utmost importance and priority in confirming a clinical suspicion, i.e. including (ruling in) or excluding (ruling out) a clinical diagnosis. In this respect, Point-of-Care Testing (POCT) is extremely important in case of emergency medical health care particularly. POCT is a diagnostic laboratory testing service performed in a number of different clinical settings such as emergency room, admission unit, operating room, intensive care unit (ICU), outpatient clinic, ward, etc1,2. However, analytical and technological requirements in terms of instrumentation, manpower (medical biochemists, medical technologists, others) and logistics must be considered carefully. The evolution of small portable POCT devices/instruments that measure electrolytes, blood gases, and other emergency analyses has helped immensely to set up POCT facilities. In many hospitals, POCT has become an integral part of diagnostic services, thus saving lives in ICU and emergencies specially2,3.

POCT introduces a range of issues such as management and visibility of remote testing site, data collection, quality control regime, analyzer status and the use of the equipment. Analysis by certified personnel, resources required to deliver an extended diagnostic service while maintaining the level of quality must be considered adequately1,2. The Royal College of Pathologists in 2004 stated that"a modern pathology service will need to consider a wide range of technologies to provide its service, and this may be achieved by linking disseminated laboratory services as part of networked area with information technology (IT) connection (e.g. by linking POCT systems remotely with central core laboratories)"2,3. A fully functional POCT connectivity network could also address the points raised in the POCT guidelines produced by the Institute of Biomedical Science, which indicates that "It is imperative that wherever POCT is operated that it is monitored and supervised by qualified staff of a Clinical Pathology Accreditation (CPA) accredited clinical laboratory" 4,5 .

The evolving technological advances that enabled the development of novel POCT instruments and the underlying analytical techniques are glucose biosensor strips and lateral flow strips using immobilized antibodies to determine a range of parameters including cardiac markers and infectious pathogens. The second category of devices is larger, often bench-top devices which are essentially laboratory instruments which have been reduced in both size and complexity. These include critical care unit (CCU) analyzers and, more recently, small hematology and immunology analysers 5,6. New emerging devices include those that are utilizing molecular techniques such as PCR to provide infectious disease testing in a sufficiently small device to be used at the point of care. This area is likely to grow with many devices being developed and likely to reach the commercial market in the next 
44 Bangladesh J Med Biochem 2017; 10(2)

few years ${ }^{6}$. Additionally, it is very desirable to measure various analytes simultaneously in the same specimen, allowing a rapid, low-cost, and reliable quantification. Therefore, multiplexed point-of-care testing (xPOCT) has become more important for medical diagnostics in the last decade. POCT has become established worldwide and finds vital roles also in public health 7 .

The main advantages and potential operational benefits of POCT are more rapid clinical decision making, reduced operating time, reduced high-dependence, reduced postoperative care time, reduced emergency room time, reduced number of hospital beds required, reduced risk of results being lost, ensured optimal use of professional time and finally improved health outcomes ${ }^{7}$. With more and more understanding on the biochemical and molecular basis of illnesses, the discipline of Medical Biochemistry played in the past, is playing presently and will play in the future as well increasingly important roles in the diagnosis, management and prevention of various human diseases. Bangladesh, being a digital country, may thus adapt POCT introducing the system at the district level with IT connections to render efficient and effective laboratory diagnostic services up to remote Upazilla level communities.
ASM Giasuddin

\section{References}

1. Price CP, John AS. Point-of- Care instrumentation. In: Burtis CA, Bruns DE (Editors). Tietz Fundamentals of Clinical Chemistry and Molecular Diagnostics, Seventh Edition; St. Louis, USA: Elsevier Saunders; 2015: 272-85.

2. The Royal College of Pathologist Guidelines on point-of- care testing. 2004.www. rcpath.org/ ewsources/pdf/ Point-of- Care Testing-updated Oct 04-pdf.

3. Sladen A. Point of Care testing and IT connectivity. Point of Care Testing, March 2009. www.menarini.com/diagnostics.

4. Institute of Biomedical Science Point-of- care testing (near-patient testing). Guidance of the involvement of the clinical laboratory. 2004. (www.ibms.org)

5. Point-of- Care testing- Wikipedia. http://en. Wikipedia. org/wiki/ Point-of-care testing. (Accessed 01 April 2018).

6. Existing and Emerging Technologies for Point-of Care Testing. https:// www.ncbi.nlm.nih. gov/pmc/ articles/ PMC4204237. (Accessed 01 April 2018).

7. Point-of- Care testing (POCT): Current techniques and futureperspectives. https://www. sciencedirect.com/science/ article/pii/ S0165993611000860. (Accessed 01 April 2018). 